\title{
Preface: nature inspired solutions for high performance computing
}

\author{
Gianluigi Folino • Carlo Mastroianni • \\ Sanaz Mostaghim
}

Published online: 13 April 2012

(C) Springer Science+Business Media B.V. 2012

Computer systems are characterized by an ever-growing complexity and a pronounced distributed nature. Since controlling highly distributed systems and managing the communication among them are far beyond the capabilities of a central entity, it is essential to develop new decentralized architectures. Such architectures, for example Grids, Clouds and P2P systems, are increasingly popular, but they need new types of algorithms to be efficiently managed.

The recent paradigm of Organic computing (OC) concerns a large collection of intelligent (embedded) systems with potentially unlimited networks and spontaneous local interactions (Merkle et al. 2008; Müller-Schloer et al. 2011). The goal of OC is to develop new concepts and tools to manage the complexity of such systems, which must be designed to be adaptive, self-organized and manageable at the same time (Schmeck 2005). Adaptive OC systems must be capable of learning and adjusting their behaviour to dynamically changing environments. In addition, they should have self-x properties such as self-healing, selfdefining, self-configuring and self-optimizing. These properties are inspired by the mechanisms used by the autonomic nervous system to regulate the body without conscious input from the individual. Bio-inspired algorithms and techniques feature fault-tolerant and self-adaptive behaviours that help to boost the autonomic nature of

G. Folino $(\bowtie) \cdot$ C. Mastroianni

ICAR-CNR, Rendi, CS, Italy

e-mail: folino@icar.cnr.it

C. Mastroianni

e-mail: mastroianni@icar.cnr.it

S. Mostaghim

Karlsruhe Institute of Technology (KIT), Karlsruhe, Germany

e-mail: sanaz.mostaghim@kit.edu distributed systems (Germain-Renaud and Rana 2009), and are proving effective to solve highly parallel and distributed problems. These techniques sometimes rely on the operations of agents, whose behaviour is inspired by biological systems, including ant colonies, bird flocks, honey bees, bacteria, and many more. In such systems, "swarm intelligence" emerges from the interaction of a large number of very simple agents.

Bio-inspired algorithms have been successfully used as alternative and/or superior solutions to the problems that traditional algorithms cannot solve satisfactorily. In recognition of their achievements and potential, bio-inspired algorithms were named one of Scientific American magazine's 10 "World Changing Ideas 2010". Bio-inspired algorithms and systems are applied to hard and large problems in a variety of areas. Some examples are optimization problems solved with genetic algorithms, routing strategies inspired by honey bee behaviour, ant-inspired resource discovery and data mining computations in Grid, Cloud and P2P frameworks, and so on.

The papers included in this special issue show recent results in the area of bio-inspired distributed computing. The authors presented their preliminary works in the 3rd Workshop on Bio-Inspired and Self-* Algorithms for Distributed Systems, BADS 2011 (Folino et al. 2011), which was held together with the International Conference on Autonomic Computing (ICAC 2011) in Karlsruhe, Germany, in June 2011. The three papers were first selected among the papers presented in BADS 2011; then, they were extended and refined through two rounds of reviews.

The paper "Protein structure prediction using distributed parallel particle swarm optimization", by Ivan Kondov, demonstrates the efficiency of the standard Particle swarm optimization (PSO) algorithm in the hard task of finding 
the folded state of two proteins starting from completely extended conformations. Experimental results confirm that the predicted structure of the larger protein is in good agreement with the structure provided from the Protein Data Bank. The advantage of the distributed approach consists in the capacity to speed up the simulation linearly with the number of workers and to considerably reduce the prediction time without loss of accuracy.

In the paper "Description and composition of bioinspired design patterns: a complete overview", by Jose Luis Fernandez Marquez et al., the authors observe that bio-inspired self-organizing mechanisms have been applied to different domains, achieving results beyond traditional approaches, and intend to respond to the needs for an ensemble overview of such mechanisms in terms of modular and reusable design patterns organized into different layers. The paper provides additional hints for selecting and using the appropriate design pattern for any specific application domain.

The paper "The grid, the load and the gradient: a bioinspired approach to load balancing", by Amos Brocco, presents a fully distributed load balancing mechanism, called Ozmos, which aims at increasing the efficiency of distributed computing systems through peer-to-peer interaction between nodes. The algorithm exploits the work of ant-like agents, which spread information among the nodes of a Chord-like overlay and reschedule tasks from overloaded nodes to lightly loaded ones. The performance results reported in the paper show that the approach allows the load to be effectively balanced, both with homogeneous and heterogeneous resources.
Although it was not explicitly planned for, all contributions involved young researchers in or just after their $\mathrm{PhD}$ studies. This is yet another indication of the liveliness and potential of the field and generates prospects of interesting results to be produced in the future.

Acknowledgments The authors are grateful to the Editor-in-chief, G. Rozenberg, and to the Associate Editor, J. N. Kok, for giving us the opportunity to publish this special issue and for his support in the editing process, to the reviewers whose critical comments and suggestions helped to ensure a high quality volume and, of course, to the authors for their willingness to participate in this endeavour and their patience in complying with editors' and reviewers' requests.

\section{References}

Folino G, Mastroianni C, Mostaghim S, Suzuki J (eds) (2011) BADS '11: proceedings of the 3rd workshop on bio-inspired and self-* algorithms for distributed systems. ACM Press, Karlsruhe

Germain-Renaud C, Rana OF (2009) The convergence of clouds, grids, and autonomics. IEEE Internet Comput 13(6):9

ICAC (2011) In: Schmeck H, Rosenstiel W, Abdelzaher TF, Hellerstein J (eds) Proceedings of the 8th International Conference on Autonomic Computing, Karlsruhe, Germany

Merkle D, Middendorf M, Scheidler A (2008) Organic computing and swarm intelligence. In: Blum C, Merkle D (eds) Swarm intelligence: introduction and applications. Natural computing series. Springer, New york, pp 253-281

Müller-Schloer C, Schmeck H, Ungerer T (eds) (2011) Organic computing: a paradigm shift for complex systems. Springer, Basel

Schmeck H (2005) Organic computing: a new vision for distributed embedded systems. In: Proceedings of the 8th IEEE international symposium on object-oriented real-time distributed computing (ISORC'05), pp 201-203 